\title{
The Optimal Portfolio Model Based on Mean-CVaR
}

\author{
Xing Yu*, Hongguo Sun, Guohua Chen \\ Department of Mathematics \& Applied Mathematics, \\ Humanities \& Science and Technology Institute of Hunan, Loudi, China \\ E-mail: "hnyuxing@163.com
}

Received September 4, 2011; revised October 5, 2011; accepted October 15, 2011

\begin{abstract}
This paper proposed the optimal portfolio model maximizing returns and minimizing the risk expressed as CvaR under the assumption that the portfolio yield subject to heavy tail. We use fuzzy mathematics method to solve the multi-objectives model, and compare the model results to the case under the normal distribution yield assumption based on the portfolio VaR through empirical research. It is showed that our return is approximate to $\mathrm{M}-\mathrm{V}$ model but risk is higher than $\mathrm{M}-\mathrm{V}$ model. So it is illustrated that $\mathrm{CVaR}$ predicts the potential risk of the portfolio, which will help investors to cautious investment.
\end{abstract}

Keywords: The Optimal Portfolio, VaR, CVaR, Multi-Objectives Programming, Fuzzy Mathematics Method

\section{Introduction}

Portfolio optimization has come a long way from Markowitz (1952) [1] seminal work which introduces return/variance risk management framework, which is called $\mathrm{M}-\mathrm{V}$ model. There is a lot of literature has improved the $\mathrm{M}-\mathrm{V}$ model. One line of work has focused on the assumption that portfolio return with normal distribution, but there is substantial empirical evidence that financial returns exhibit fat-tails and excess kurtosis after accounting for the clustering of volatility and autocorrelation. Using different approaches to the problem and different sets of data, these studies consistently find high kurtosis and heavy tails different models have been suggested to explain these empirical facts. Mandelbrot [2] and Fama [3] proposed the stable Paretian distribution which was later incorporated as a building block in GARCH-type processes, see for example Mittnik et al. [4] and Mittnik and Paolella [5]. Another line of work has focused on developing more realistic models of changes in risk factors. As a supplement (or alternative) to $\mathrm{VaR}$, another percentile risk measure which is called Conditional Value-at-Risk. (CVaR), which is defined as the conditional expected loss under the condition that it exceeds VaR, see Rockafellar and Uryasev [6]. It has been shown (Pflug, [7]) that CVaR is a coherent risk measure that has many attractive properties including convexity, e.g., see Ogryczak and Ruszczynski [8] for an overview of CVaR. In addition, minimizing CVaR typically leads to a portfolio with a small VaR.

This paper contributes to both lines of investigation by developing methods for calculating portfolio maximizing returns and minimizing the risk expressed as CVaR under the assumption that the portfolio yield subject to heavy tail distribution under discrete case. This paper is organized as follows: In Section 2, the notions of CVaR is introduced. In Section 3, the optimal portfolio model is proposed. In Section 4, empirical study is performed and compared the result to the case of Mean-variance model.

\section{Conditional Value-at-Risk}

The approach developed in (Rockafellar and Uryasev, [6]) provides the foundation for the analysis conducted in this paper. For each investment share $w$, the loss $f(w, y)$ is a random variable having a distribution induced by that of $y$. For a portfolio $w$, the loss is usually defined $f(w, y)=-w^{T} y$, given a believe degree $\beta(0<\beta<1)$, $a R_{\beta}(w), \mathrm{CVaR}_{\beta}(w)$ are defined as [7]:

$$
\begin{gathered}
\operatorname{VVaR}_{\beta}(w)=\min \left\{\alpha \mid \int_{f(w, y) \leq \alpha} p(y) \mathrm{d} y \geq \beta\right\} \\
\mathrm{CVaR}_{\beta}(w)=(1-\beta)^{-1} \int_{f(w, y) \geq \operatorname{VaR}_{\beta}(x)} f(w, y) p(y) \mathrm{d} y(2)
\end{gathered}
$$

where $p(y)$ is the p.d.f of random $y$, in our paper, $p(y)$ is a heavy tail distribution, for example multi t distribution, not a normal distribution. In this case, the density function usually complex, so that it is more complex to calculate $\operatorname{VaR}_{\beta}(w), \mathrm{CVaR}_{\beta}(w)$ using formula (1) (2). In order to overcome the difficulties, [9] defined a new simpler function under discrete case: 


$$
\tilde{F}_{\beta}(w, \alpha)=\alpha+\frac{1}{m(1-\beta)} \sum_{k=1}^{m}\left[f\left(w, y_{k}\right)-\alpha\right]^{+}
$$

where the sampling generates a collection of vectors $y_{1}, y_{2} \cdots y_{m}$, the model of $\operatorname{minCVaR}_{\beta}(w)$ is equal to deal with the linear programming $(P 1)$ :

$$
\begin{array}{r}
\min \alpha+\frac{1}{m(1-\beta)} \sum_{k=1}^{m} u_{k} \\
(P 1)\left\{\begin{array}{c}
\text { s.t } w^{T} y+\alpha+u_{k} \geq 0 \\
u_{k} \geq 0 \\
\sum_{i=1}^{n} w_{i}=1 \\
0 \leq w_{i} \leq 1
\end{array}\right.
\end{array}
$$

\section{Solution to a General Fuzzy Multi-Objective Model}

\subsection{The Multi-Objective Model}

Let

$$
A=\left(a_{i j}\right)_{m \times n}, B=\left(c_{i j}\right)_{r \times n}, b=\left(b_{1}, b_{2} \cdots b_{m}\right)^{T}
$$$$
x=\left(x_{1}, x_{2} \cdots x_{m}\right)^{T}, Z=\left(Z_{1}, Z_{2} \cdots Z_{r}\right)^{T},
$$

a multi-objective fuzzy programming is described as:

$$
\begin{aligned}
& \max Z=C x \\
& \text { s.t }\left\{\begin{array}{c}
A x \leq b \\
x \geq 0
\end{array}\right.
\end{aligned}
$$

Because the objective functions of multi-objective programming are more than one, it is difficult to reach a certain point for all of the objective functions, to whose maximum, that is the optimal solution is usually does not exist. Therefore, it needs to make a compromise plan making each target function as large as possible in a specific problem. And fuzzy mathematical programming method can deal with the problem, which will turn the multi-target model to a single one.

\subsection{Solution of Multi-Objective Linear Programming Model with Fuzzy Mathematics}

Step 1: to solve every single maximum objective

$$
Z_{i}, i=1,2 \cdots r
$$

under the constrains $\left(1^{\prime}\right),\left(2^{\prime}\right)$,

$$
Z_{i}^{*}=\max \left(Z_{i} \mid Z_{i}=\sum_{j=1}^{n} c_{i j}, A x \leq b, x \geq 0\right), i=1,2 \cdots r .
$$

To choose $d_{i},\left(d_{i}>0\right)$ as the corresponding fuzzy telescopic factor for each target $Z_{i}, i=1,2 \cdots r$, Generally, fuzzy telescopic factors are chosen according to various sub-targets importance, that is the more important goal, the smaller the flexible index should be. In this paper, let

$$
d_{i}=Z_{i}^{*}-Z_{i}^{-},
$$

where $d Z_{i}^{-}=\min Z_{i}$, which is solved following the same method as $Z_{i}^{*}$.

Step 2: constructing fuzzy objective of $\tilde{M}_{i}$ target $Z_{i}$, whose membership function is:

$$
\begin{aligned}
& \tilde{M}_{i}(x)=g_{i}\left(\sum_{j=1}^{n} c_{i j} x_{j}\right) \\
& =\left\{\begin{array}{c}
0, \sum_{j=1}^{n} c_{i j} x_{j}<Z_{i}^{*}-d_{i} \\
1-\frac{1}{d_{i}}\left(Z_{i}^{*}-\sum_{j=1}^{n} c_{i j} x_{j}\right), Z_{i}^{*}-d_{i} \leq \sum_{j=1}^{n} c_{i j} x_{j}<Z_{i}^{*} \\
1, Z_{i}^{*} \leq \sum_{j=1}^{n} c_{i j} x_{j}
\end{array}\right. \\
& \text { Let } \quad \tilde{M}(x)=\bigcap_{i=1}^{r} \tilde{M}_{i}(x),
\end{aligned}
$$

the multi-objective problem $P 2$ is transformed as:

$$
\left\{\begin{array}{c}
\max Z=\lambda \\
1-\frac{1}{d_{i}}\left(Z_{i}^{*}-\sum_{j=1}^{n} c_{i j} x_{j}\right) \geq \lambda, i=1,2 \cdots r \\
\sum_{j=1}^{n} a_{k j} x_{j} \leq b_{k}, k=1,2 \cdots m \\
\lambda \geq 0, x_{1}, x_{2} \cdots x_{n} \geq 0
\end{array}\right.
$$

This is a single objective linear programming solved with LINGO easily.

\section{The Optimal Portfolio Model}

For investors, the aim is to seek the maximize returns while controlling risk as minimal risk.Suppose that the returns of portfolio follows heavy tail distribution, such as multivariate $\mathrm{t}$ distribution.We propose the optimal portfolio under Mean-CVaR model:

$$
\text { (P4) }\left\{\begin{array}{l}
\max \quad E(R)=w^{T} \mu \\
\min \quad \operatorname{CVaR}(w) \\
\text { s.t } \sum_{i=1}^{n} w_{i}=1 \\
0 \leq w_{i} \leq 1
\end{array}\right.
$$

$\operatorname{Model}(P 4)$ is a multi-objective optimization problem,it is can be solved by fuzzy mathematics method. 


\section{Empirical Study}

\subsection{Mean-CVaR Model under Heavy Distribution}

We choose two stocks (MS and Google), date begins 2008.1.1 to 2011.8.26, with 922 closed days, calculate each day yield:

$$
y_{i, j}=\frac{p_{i, j}-p_{i, j-1}}{p_{i, j-1}},
$$

where $p_{i, j}$ is previous day's closing price and $p_{i, j-1}$ is the day after. And we also can get the average yield of MS is $\mu_{1}=0.0001789$, kurtosis is 70.288 ; the average yield of Google is $\mu_{2}=0.0000044$, kurtosis is 9.693, compare to normal return's kurtosis 3, the two stocks returns's is larger. That is, the return is heavy tails.

To solve model $(P 4)$, let $m=922, \alpha=0.01$, we solve the following sub-model using LINGO:

$$
\begin{gathered}
(P 5)\left\{\begin{array}{c}
\max E(R)=w^{T} \mu \\
\text { s.t } \sum_{i=1}^{n} w_{i}=1 \\
0 \leq w_{i} \leq 1
\end{array}\right. \\
\left(P 5^{\prime}\right)\left\{\begin{array}{c}
\min E(R)=w^{T} \mu \\
\text { s.t } \sum_{i=1}^{n} w_{i}=1 \\
0 \leq w_{i} \leq 1
\end{array}\right.
\end{gathered}
$$

We get $R^{+}=\max E(R), R^{-}=\min E(R)$.

The next step is to solve sub-model $(P 6)$ and $\left(P 6^{\prime}\right)$

$$
\begin{aligned}
& (P 6)\left\{\begin{array}{c}
\min \operatorname{CVaR}(w) \\
\text { s.t } \sum_{i=1}^{n} w_{i}=1 \\
0 \leq w_{i} \leq 1
\end{array}\right. \\
& \left(P 6^{\prime}\right)\left\{\begin{array}{c}
\max \operatorname{CVaR}(w) \\
\text { s.t } \sum_{i=1}^{n} w_{i}=1 \\
0 \leq w_{i} \leq 1
\end{array}\right.
\end{aligned}
$$

We get: $C^{+}=\max \operatorname{CVaR}(w) \quad C^{-}=\min \operatorname{CVaR}(w)$ At last, we solve problem $(P 7)$ to get the optimal portfolio: $\max \lambda$

$$
\text { s.t }\left\{\begin{array}{c}
E(R)-\left(R^{+}-R^{-}\right) \lambda \geq R^{-} \\
\operatorname{CVaR}(w)-\left(C^{+}-C^{-}\right) \lambda \leq C^{+} \\
\sum_{i=1}^{n} w_{i}=1 \\
0 \leq w_{i} \leq 1 \\
\lambda \geq 0
\end{array}\right.
$$

We get the optimal portfolio is $w=[1,0]$, in this case, $\max E(w)=0.0001789, \min \operatorname{CVaR}(w)=0.020849$.

\subsection{Mean-VaR Model under Normal Distribution}

We can also solve Markowitz model, and get the optimal portfolio is $w=[0.99,001]$, in which case,

$$
\max E(w)=0.0001789, \quad \min \operatorname{VaR}(w)=0.003
$$

It is showed that our max return is equal to and risk is higher than M-V model. So the CVaR predicts the potential risk of the portfolio, which help investors cautious investment.

Although the empirical research only include two stocks, but it is easy to be extended to several stocks case.

\section{References}

[1] H. M. Markowitz, "Portfolio Selection," Journal of Finance, Vol. 7, No. 1, 1952, pp. 77-91. doi:10.2307/2975974

[2] B. Mandelbrot, "The Variation of Certain Speculative Prices,” Journal of Business, Vol. 26, No. 4, 1963, pp. 394-419. doi:10.1086/294632

[3] E. Fama, “The Behavior of Stock Market Pricess,” Journal of Business, Vol. 38, No. 1, 1965, pp. 34-105. doi:10.1086/294743

[4] S. Mittnik, M. Paolella and S. Rachev, "Stationarity of Stable Power-GARCH Processes," Journal of Econometrics, Vol. 106, No. 1, 2002, pp. 97-107. doi:10.1016/S0304-4076(01)00089-6

[5] S. Mittnik and M. Paolella, "Prediction of Fnancial downside-Risk with Heavy-Tailed Conditional Distributions," In: S. T. Rachev, Ed., Handbook of Heavy Tailed Distributions in Finance, Elsevier, New York, 2003, pp. 385404. doi:10.1016/B978-044450896-6.50011-X

[6] R. T. Rockafellar and S. Uryasev, "Optimization of Conditional Value-at-Risk,” Journal of Risk, Vol. 2, 2000, pp. 21-41.

[7] G. Ch. Pflug, "Some Remarks on the Value-At-Risk and the Conditional Value-At-Risk,” In: S. Uryasev, Ed., Probabilistic Constrained Optimization: Methodology and Applications, Kluwer Academic Publishers, Dordre- cht, 2000.

[8] W. Ogryczak and A. Ruszczynski, "Dual Stochastic DomiNance and Quantile Risk Measures," International Transactions in Operational Research, Vol. 9, No. 5, 2002, pp. 661-680. doi:10.1111/1475-3995.00380

[9] S. Uryasev, "Conditional Value-at-Risk: Optimization Algorithms and Applications," Proceedings of the 2000 Conference on Computational Intelligence for Financial Engineering, New York, 26-28 March 2000, pp. 49-57. 